\title{
On the new modes of planetary-scale electromagnetic waves in the ionosphere
}

\author{
G. D. Aburjania ${ }^{1}$, K. Z. Chargazia ${ }^{1}$, G. V. Jandieri ${ }^{2}$, A. G. Khantadze ${ }^{3}$, and O. A. Kharshiladze ${ }^{4}$ \\ ${ }^{1}$ I. Vekua Institute of Applied Mathematics, Laboratory of Investigation of Extraordinary Phenomena, Tbilisi State University, \\ University Str., 2, 0143 Tbilisi, Georgia \\ ${ }^{2}$ Georgian Technical University, Department of Physics, Kostava Str., 77, 0175 Tbilisi, Georgia \\ ${ }^{3}$ Chair of Meteorology and Hydrology, Tbilisi State University, Chavchavadze Ave., 1, 0175 Tbilisi, Georgia \\ ${ }^{4}$ Tbilisi State University, Faculty of Physics, Chavchavadze Ave., 3, 0128 Tbilisi, Georgia
}

Received: 18 October 2002 - Revised: 21 April 2003 - Accepted: 22 August 2003 - Published: 2 April 2004

\begin{abstract}
Using an analogy method the frequencies of new modes of the electromagnetic planetary-scale waves (with a wavelength of $10^{3} \mathrm{~km}$ or more), having a weather forming nature, are found at different ionospheric altitudes. This method gives the possibility to determine spectra of ionospheric electromagnetic perturbations directly from the dynamic equations without solving the general dispersion equation. It is shown that the permanently acting factor-latitude variation of the geomagnetic field generates fast and slow weakly damping planetary electromagnetic waves in both the E- and F-layers of the ionosphere. The waves propagate eastward and westward along the parallels. The fast waves have phase velocities (1-5) $\mathrm{km} \mathrm{s}^{-1}$ and frequencies $\left(10^{-1}\right.$ $10^{-4}$ ), and the slow waves propagate with velocities of the local winds with frequencies $\left(10^{-4}-10^{-6}\right) \mathrm{s}^{-1}$ and are generated in the E-region of the ionosphere. Fast waves having phase velocities (10-1500) $\mathrm{km} \mathrm{s}^{-1}$ and frequencies (1$\left.10^{-3}\right) \mathrm{s}^{-1}$ are generated in the F-region of the ionosphere. The waves generate the geomagnetic pulsations of the order of one hundred nanoTesla by magnitude. The properties and parameters of the theoretically studied electromagnetic waves agree with those of large-scale ultra-low frequency perturbations observed experimentally in the ionosphere.
\end{abstract}

Key words. Ionosphere (ionospheric disturbances; waves propagation; ionosphere atmosphere interactions)

\section{Introduction}

Numerous ground-based and satellite observations show that the background global planetary-scale electromagnetic wavy perturbations $\left(\geq 10^{3} \mathrm{~km}\right)$ regularly exist in the ionosphere at any season of the year. The observations verify (Sharadze et al., 1989; Cavalieri et al., 1974) the presence of slow (with phase velocities equal to local winds velocities), longperiod (a few days and more) and large-scale waves (with

Correspondence to: G. D. Aburjania

(aburj@viam.hepi.edu.ge) wavelength $\lambda \sim 10^{3}-10^{4} \mathrm{~km}$ ) in the E-layer of the ionosphere. Different from the usual weather forming planetary Rossby waves, they cause substantial disturbances of the geomagnetic field (up to ten nanotesla (nT)). Ionospheric observations at the middle latitudes of the E-layer verify the existence of fast, large-scale electromagnetic perturbations, too (Bauer et al., 1995; Sharadze et al., 1988). They propagate along the latitude circles of the Earth with velocities from a few hundred $\mathrm{m} \mathrm{s}^{-1}$ to a few tens of $\mathrm{km} \mathrm{s}^{-1}$. Their periods vary in the interval from a few minutes to a few hours, with a wavelength of the order of a $\mathrm{km}$ or more, and an amplitude of tens of hundred nT. The phase velocities of these perturbations differ by magnitude at daily and nightly conditions in the E-layer of the ionosphere.

These waves have mainly zonal character and are revealed especially during magnetic storms and sub-storms (Hajkowicz, 1991), earthquakes (Hayakawa, 1999), artificial explosions (Al'perovich et al., 1985) and so on. They play an important role in the large-scale synoptic processes and give the possibility of obtaining valuable information about external sources and dynamical processes, which take place in the ionosphere during this period.

Thus, the main problem is to find the factors which generate the background planetary-scale electromagnetic waves in the different layers of the ionosphere. It will be shown below that the weather forming planetary electromagnetic waves exist due to latitude inhomogeneity of the geomagnetic field in the ionosphere.

\section{Formulation of the problem and basic equations}

The ionosphere represents partially ionized triple component plasma. To describe it we take quasi-hydrodynamic equations, which differ from hydrodynamic equations by the presence of "friction force", caused by a collision of different particles (Cowling, 1975; Alfvén and Falthammar 1963; Wait, 1962). Quasi-hydrodynamic equations describe the flows, electromagnetic currents and all diffusive processes in the 
ionospheric plasma. However, the diffusive processes, compressibility and inhomogeneity of the atmosphere play a secondary role for considered large-scale ionospheric perturbations (wavelength $\lambda \geq 10^{3} \mathrm{~km}$ ). Thus, we can substantially simplify these equations and obtain the following set of equations (Gershman, 1974; Cowling, 1975; Khantadze 1973; Wait 1962):

$$
\begin{gathered}
\rho_{n} \frac{\partial \mathbf{V}_{n}}{\partial t}=\mathbf{F}_{n}-\rho_{i} v_{i n}\left(\mathbf{V}_{n}-\mathbf{V}_{i}\right)-\rho_{e} v_{e n}\left(\mathbf{V}_{n}-\mathbf{V}_{e}\right), \\
\rho_{e} \frac{\partial \mathbf{V}_{e}}{\partial t}=\mathbf{F}_{e}-\rho_{e} v_{e n}\left(\mathbf{V}_{e}-\mathbf{V}_{n}\right)- \\
\rho_{e} v_{e i}\left(\mathbf{V}_{e}-\mathbf{V}_{i}\right)-\mathrm{eNE}-\frac{\mathrm{eN}}{\mathrm{c}} \mathbf{V}_{e} \times \mathbf{H}_{0}, \\
\rho_{i} \frac{\partial \mathbf{V}_{i}}{\partial t}=\mathbf{F}_{i}-\rho_{i} v_{i n}\left(\mathbf{V}_{i}-\mathbf{V}_{n}\right)- \\
\rho_{e} v_{e i}\left(\mathbf{V}_{i}-\mathbf{V}_{e}\right)+\mathrm{eNE}+\frac{\mathrm{eN}}{\mathrm{c}} \mathbf{V}_{i} \times \mathbf{H}_{0}, \\
\nabla \cdot \mathbf{V}_{n}=0, \quad \nabla \cdot \mathbf{V}_{e}=0, \quad \nabla \cdot \mathbf{V}_{i}=0 .
\end{gathered}
$$

Here, indices $n, e$ and $i$ denote molecules (neutral particles), electrons and ions; $\mathbf{V}$ is velocity; $\rho_{n}=\mathrm{N}_{n} \mathrm{M}, \rho_{e}=\mathrm{Nm}$, $\rho_{i}=\mathrm{NM}$ are densities; $\mathrm{m}$ and $\mathrm{M}$ are masses of electrons and ions (molecule), respectively; $\mathrm{N}_{n}$ and $\mathrm{N}$ denote concentrations of the neutral particles and plasma; $\mathrm{c}$ is a light speed; $v_{e i}, v_{e n}, v_{i n}$ denote frequencies of collision of electrons with ions and molecules, of ions with molecules, respectively; $\mathbf{E}$ is the strength of the induced electric field; $\mathbf{H}_{0}$ is the strength of the geomagnetic field; $\mathbf{F}_{n}, \mathbf{F}_{e}, \mathbf{F}_{i}$ denote the nonelectromagnetic forces containing gradients of impulse flux density tensor in general case; $\nabla(\partial / \partial x, \partial / \partial y, \partial / \partial z)$, is the nabla operator.

Equations (1)-(4), state and thermal equations and Maxwell's equations form a close system for each component. For simplification of these equations we take into account the results of experimental observations of the dynamical processes.

In the ionosphere at a height $(80-500) \mathrm{km}$ $\left(\eta=\mathrm{N} / \mathrm{N}_{n} \sim 10^{-9}-10^{-4} \ll 1\right) \quad$ non-electromagnetic forces $\mathbf{F}_{n}, \mathbf{F}_{e}, \mathbf{F}_{i}$ are proportional to the densities of medium components and, hence, $\eta \ll 1,\left|\mathbf{F}_{i} \leq\right| \mathbf{F}_{e}|\ll| \mathbf{F}_{n} \mid$. So, $\mathbf{F}_{e}$ and $\mathbf{F}_{i}$ cannot induce big currents. The inertia of electrons and ions can be neglected comparing with inertia of the neutral particles. Taking into account all of these circumstances in Eqs. (1)-(4), we obtain the equation of ionospheric medium motion:

$\rho_{n} \frac{\partial \mathbf{V}_{n}}{\partial t}=\mathbf{F}_{n}+\frac{1}{\mathrm{c}} \mathbf{j} \times \mathbf{H}_{0}$,

where $\mathbf{j}=\mathrm{eN}\left(\mathbf{V}_{i}-\mathbf{V}_{e}\right)$ is the density of current. Equations (2) and (3) may be rewritten as

$$
\begin{aligned}
& -\frac{v_{e n}}{\omega_{e}}\left(\mathbf{V}_{e}-\mathbf{V}_{n}\right)-\frac{\nu_{e i}}{\omega_{e}}\left(\mathbf{V}_{e}-\mathbf{V}_{i}\right)+\mathbf{V}_{D} \times \mathbf{h}_{0}=\mathbf{V}_{e} \times \mathbf{h}_{0}, \\
& -\frac{\nu_{i n}}{\omega_{i}}\left(\mathbf{V}_{i}-\mathbf{V}_{n}\right)-\frac{\nu_{e i}}{\omega_{e}}\left(\mathbf{V}_{i}-\mathbf{V}_{e}\right)+\mathbf{V}_{i} \times \mathbf{h}_{0}=\mathbf{V}_{D} \times \mathbf{h}_{0},
\end{aligned}
$$

where $\omega_{e}=\mathrm{eH}_{0} / \mathrm{mc}$ and $\omega_{i}=\mathrm{eH}_{0} / \mathrm{Mc}$ denote cyclotron frequencies of electrons and ions, respectively, $\mathbf{V}_{D}=\mathrm{c} \mathbf{E} \times \mathbf{H}_{0} / \mathrm{H}_{0}^{2}$ is the electron drift velocity; $\mathbf{h}_{0}=\mathbf{H}_{0} / \mathrm{H}_{0}$ is the unit vector along the strength of the geomagnetic field. In the ionosphere $\omega_{e} \approx 10^{7} \mathrm{~s}^{-1}, \omega_{i} \approx(1.5-3) \times 10^{2} \mathrm{~s}^{-1}$, the collision frequency reaches its maximal value $v_{e i} \approx 10^{4} \mathrm{~s}^{-1}$, $v_{i n} \approx 10^{4} \mathrm{~s}^{-1}, v_{e n} \approx 10^{5} \mathrm{~s}^{-1}$ at heights $(80-500) \mathrm{km}$ in the lower layer of the ionosphere and quickly decreases in proportion to height. Thus, we can conclude that $v_{e i} / \omega_{e} \ll 1$, $v_{e n} / \omega_{e} \ll 1$ in the E- and F-layers of the ionosphere. It means that the electron component of the ionospheric plasma is always magnetized in this region of the upper atmosphere. Taking into account these inequalities, Eqs. (6) and (7) can be reduced to the following form:

$$
\begin{aligned}
& \mathbf{V}_{D} \times \mathbf{h}_{0}=\mathbf{V}_{e} \times \mathbf{h}_{0} \Rightarrow \mathbf{V}_{e}=\mathbf{V}_{D} \Rightarrow \mathbf{E}=-\frac{1}{\mathrm{c}} \mathbf{V}_{e} \times \mathbf{H}_{0}, \\
& \mathbf{V}_{i}=\mathbf{V}_{n}+\mathbf{j} \times \mathbf{H}_{0} /\left(\rho \mathrm{c} v_{i}\right), \quad v_{i}=\mathrm{N} v_{i n} / \mathrm{N}_{n} .
\end{aligned}
$$

Therefore, in the E- and F-layers of the ionosphere electrons move with electron drift velocity $\left(\mathbf{V}_{e}=\mathbf{V}_{D}\right)$ and the electrons are frozen into the geomagnetic field $\mathbf{H}_{0}\left(\partial \mathbf{h} / \partial t=\nabla \times \mathbf{V}_{e} \times \mathbf{H}_{0}\right)$, h denotes the perturbation of the geomagnetic field.

Multiplying the Eq. (8) by $\mathbf{H}_{0}$, we obtain an important equality $\mathbf{E} \cdot \mathbf{H}_{0}=0 \Rightarrow \mathbf{E} \perp \mathbf{H}_{0}$. This means that the strenght vector $\mathbf{E}$ of the generated internal electric field is perpendicular to the strength vector $\mathbf{H}_{0}$ of the geomagnetic field. Using Maxwell's equations we get closure of the system of Eqs. (5), (8), (9)

$$
\frac{\partial \mathbf{h}}{\partial t}=-\mathrm{c} \nabla \times \mathbf{E}, \quad \mathbf{j}=\frac{\mathrm{c}}{4 \pi} \nabla \times \mathbf{h} .
$$

Excluding E, j and using Eq. (10) and taking into account that for considered wavy processes $F_{n}=-\nabla P / \rho+\rho^{\prime} g / \rho+V \times 2 \omega_{0}$, dropping index $\mathrm{n}$ for velocity and the density of the neutral particles, we obtain the system of magneto-hydrodynamic equations for the Eand F-layers of the ionosphere:

$$
\begin{aligned}
\frac{\partial \mathbf{V}}{\partial t} & =-\frac{1}{\rho} \nabla \mathrm{P}^{\prime}+\frac{\rho^{\prime}}{\rho} \mathbf{g}+\mathbf{V} \times 2 \omega_{0}+\frac{\mathbf{F}_{A}}{\rho}, \\
\frac{\partial \mathbf{h}}{\partial t} & =\nabla \times \mathbf{V}_{e} \times \mathbf{H}_{0}= \\
& \nabla \times \mathbf{V} \times \mathbf{H}_{0}-\alpha \rho \nabla \times \frac{\mathbf{F}_{A}}{\rho}+\nabla \times \frac{1}{v_{i}} \frac{\mathbf{F}_{A}}{\rho} \times \mathbf{H}_{0},
\end{aligned}
$$

where:

$$
\begin{gathered}
\frac{\mathbf{F}_{A}}{\rho}=\frac{1}{\rho \mathrm{c}} \mathbf{j} \times \mathbf{H}_{0}=\frac{1}{4 \pi \rho} \nabla \times \mathbf{h} \times \mathbf{H}_{0} \approx \\
\mathbf{V} \times 2 \Omega_{i}-\mathbf{V}_{D} \times 2 \Omega_{i}=\mathbf{u} \times 2 \Omega_{i} ;
\end{gathered}
$$

$\sigma_{\mathrm{H}}=\mathrm{e}^{2} \mathrm{~N}\left[\omega_{\mathrm{e}} /\left(\mathrm{m}\left(\omega_{\mathrm{e}}^{2}+v_{\mathrm{e}}^{2}\right)\right)-\omega_{\mathrm{i}} /\left(\mathrm{M}\left(\omega_{\mathrm{i}}^{2}+v_{\mathrm{in}}^{2}\right)\right)\right]$ is the Hall's conductivity; Hall's parameter $\alpha$ in the general case is $\alpha=\mathrm{c}^{2} /\left(\mathrm{H}_{0} \sigma_{\mathrm{H}}\right) ; \mathrm{P}^{\prime}$ and $\rho^{\prime}$ are accordingly perturbations 
of gas-kinematic pressure and density of neutral particles; $v_{e}=v_{e i}+v_{e n} ; v_{i}=\eta v_{i n}, 2 \Omega_{\mathrm{i}}=\eta \mathrm{e} \mathbf{H}_{0} /(\mathrm{Mc})=\eta \omega_{\mathrm{i}} ; \mathbf{g}$ is the free fall acceleration; $\omega_{0}$ is the angular velocity of the Earth's rotation; $\mathbf{u}=\mathbf{V}-\mathbf{V}_{D}$. In the E-region of the ionosphere we have $\omega_{e} \gg v_{e n}, \omega_{i} \ll v_{i n}$ and $\alpha=\mathrm{c} /(\mathrm{eN})$ (Hall's conductivity disappears higher than $150 \mathrm{~km} \sigma_{H}=0$ ). From Eq. (13) it follows that the Ampere's electromagnetic force $\mathbf{F}_{A}$, acting on a unite mass of the medium $\mathbf{F}_{\mathrm{A}} / \rho=\mathbf{U} \times 2 \Omega_{i}$, has the same structure as the Coriolis acceleration $\mathbf{V} \times \mathbf{2} \omega_{0}$. Therefore, Ampere force must act on the atmospheric-ionospheric medium similar to Coriolis force. The similarity of the Ampere and Coriolis forces means that new modes of the large-scale electromagnetic oscillations must be generated due to the inhomogeneity of the geomagnetic field $\mathbf{H}$ as well as Rossby-type usual planetary waves are generated due to inhomogeneity of angular velocity of the Earth's rotation $\omega_{0}$. In this case, as it will be shown below, the first term of the electromagnetic force $\mathbf{F}_{A}$, caused by the velocity of medium motion (dynamo field $\mathbf{E}_{d}=\mathbf{V} \times \mathbf{H}_{0} / \mathrm{c}$ ), generates the slow Rossby-type electromagnetic waves; the second term of the electromagnetic force appeared due to the vortex electric field $\mathbf{E}_{V}=-\mathbf{V}_{D} \times \mathbf{H}_{0} / \mathrm{c}$ and generates the fast electromagnetic waves.

Estimations show, that for planetary scale ( $\mathrm{L} \sim\left(10^{3}-10^{4}\right) \mathrm{km}$, we shall be interested in these perturbations) in the E-region of the ionosphere with the magnetic Reynolds number $\operatorname{Re}_{m}=\omega \mathrm{L}^{2} / \nu_{H} \sim 1 / \alpha$, where $\mathrm{L}$ and $\alpha$ are the characteristic linear scale and frequency of the perturbations, and $v_{H}=\mathrm{c}^{2} /\left(4 \pi \sigma_{H}\right)$, reaches: a small enough value $\left(\operatorname{Re}_{m} \sim 20\right)$. Therefore, it is necessary to preserve Hall's term $(\sim \alpha)$ of the induction equation, but the last term of Eq. (12) can be neglected due to the condition $\sigma_{H} \gg \sigma_{\perp} \approx \sigma_{H} \omega_{i} / \nu_{i n}$ (where $\sigma_{\perp}$ is the transversal conductivity). In the F-region of the ionosphere, where Hall's effect is not important, the last term of Eq. (12) can also be neglected for planetary-scale perturbations in the first approximation as long as the Reynolds number $\operatorname{Re}_{\perp}=\omega \mathrm{L}^{2} / \nu_{\perp}\left(\nu_{\perp}=\mathrm{c}^{2} /\left(4 \pi \sigma_{\perp}\right)\right)$ is of the order of $10^{2}$. Observations show that the planetary waves propagate over great distances in the ionosphere without substantial changes (Cavalieri et al., 1976, 1974; Bauer et al., 1995; Sharadze et al., 1989, 1988). For planetary scale waves the latitude variations of the angular velocity of the Earth's rotation $\omega_{0}(\theta)$ and the geomagnetic field $\mathbf{H}_{0}\left(\theta^{\prime}\right)$ (where $\theta, \theta^{\prime}$ are geographical and geomagnetic colatitudes). Therefore, for such large-scale perturbations we must use Helmholtz equation of velocity vortex, which takes into account latitude effects of vectors $\omega_{0}$ and $\mathbf{H}_{0}$, instead of equation of motion (11), as well as dynamical meteorology (Gossard and Hooke, 1975; Holton, 1975; Khantadze, 1973). The Helmholtz equation is obtained from Eq. (11) by utilizing on both sides the operator $\operatorname{curl}=\nabla \times$. Compressibility and temperature stratification of the atmosphere, as it is mentioned above, play a secondary role for such disturbances (Pedlosky, 1979; Gossard and Hooke, 1975; Khantadze 1973).

Hence, for the E- and F-layers of the ionosphere magneto-hydrodynamic Eqs. (11)-(13) may be written in the following form:

$$
\begin{aligned}
& \frac{\partial \nabla \times \mathbf{V}}{\partial t}=\nabla \times \mathbf{V} \times 2 \omega_{0}+\frac{1}{4 \pi \rho} \nabla \times \nabla \times \mathbf{h} \times \mathbf{H}_{0}, \\
& \frac{\partial \mathbf{h}}{\partial t}=\nabla \times \mathbf{V} \times \mathbf{H}_{0}-\frac{\alpha}{4 \pi} \nabla \times \nabla \times \mathbf{h} \times \mathbf{H}_{0}, \\
& \nabla \cdot \mathbf{V}=0, \nabla \cdot \mathbf{h}=0
\end{aligned}
$$

Here, $\quad \mathbf{H}_{0}=\mathrm{H}_{0 y} \mathbf{e}_{\mathbf{y}}+\mathbf{H}_{0 z} \mathbf{e}_{\mathbf{z}}, \quad \mathrm{H}_{0 y}=-\mathrm{H}_{p} \sin \theta^{\prime}$, $\mathrm{H}_{0 z}=-2 \mathrm{H}_{p} \cos \theta^{\prime}, \mathrm{H}_{p}=3.2 \times 10^{-5} \mathrm{~T}$ is a value of geomagnetic field strength on the equator; $2 \omega_{0}=2 \omega_{0 y} \mathbf{e}_{y}+2 \omega_{0 z} \mathbf{e}_{z}$, $2 \omega_{0 y}=2 \omega_{0} \sin \theta, \quad 2 \omega_{0 z}=2 \omega_{0} \cos \theta, \quad \omega_{0}=7.3 \times 10^{-5} \mathrm{~s}^{-1}$; $\theta^{\prime}=\pi / 2-\varphi^{\prime}, \varphi^{\prime}-$ geomagnetic latitude; $\theta=\pi / 2-\varphi, \varphi-$ geographical latitude; $\mathbf{e}_{\mathbf{x}}, \mathbf{e}_{\mathbf{y}}, \mathbf{e}_{\mathbf{z}}$ denote unit vectors along the $\mathrm{x}-, \mathrm{y}-\mathrm{z}$-axes, respectively.

The closed system of Eqs. (14) and (15) contains six scalar equations and gives the possibility to calculate six unknown quantities: $\mathrm{V}_{x}, \mathrm{~V}_{y}, \mathrm{~V}_{z}, \mathrm{~h}_{x}, \mathrm{~h}_{y}, \mathrm{~h}_{z}$. After determining the values of $\mathbf{V}$ and $\mathbf{h}$, pressure $\mathrm{P}^{\prime}$ will be determined from Eq. (11) in quadrature (as far as $\rho^{\prime}=0$ ); current density and electric field are calculated from Maxwell's Eqs. (10); electron velocity is determined from the expression $\mathbf{V}_{e}=\mathbf{V}_{D}$, ion velocity is determined from Eq. (9). Thus, the initial-boundary problem of large-scale dynamics of triple component plasma for the E- and F-layers of the ionosphere in linear approximation is solved completely.

\section{Large-scale wavy perturbations}

The discussed planetary waves have a wavelength of the order of the Earth's radius $r_{0}$. Therefore, it is natural to consider the creation of large-scale perturbations in the Earth's atmosphere in a spherical coordinate system. However, the mathematical difficulties, raised by theoretical investigation of obtained equations, oblige us to consider the problem in a "standard" coordinate system (Gill, 1982; Pedlosky, 1979; Holton, 1975; Khantadze, 1973). In this system the $\mathrm{x}$-axis is directed to the east towards the parallels, the Y-axis to the north along the meridian, $\mathrm{Z}$-axis is directed vertically up (local Cartesian coordinate system). Length elements $d x, d y$, $\mathrm{dz}$ are connected with the parameters of the spherical coordinate system $\lambda, \theta, \mathrm{r}$ by the following approached formulas: $\mathrm{dx}=\mathrm{r}_{0} \sin \theta \mathrm{d} \lambda, \mathrm{dy}=-\mathrm{r}_{0} \mathrm{~d} \theta, \mathrm{dz}=\mathrm{dr}$. Velocities are equal: $\mathrm{V}_{x}=\mathrm{V}_{\lambda},-\mathrm{V}_{y}=\mathrm{V}_{\theta}, \mathrm{V}_{z}=\mathrm{V}_{r}$. Here, $\lambda$ is the longitude, $\mathrm{r}_{0}$ is the Earth's radius, $r$ is the distance from the center of the Earth along the Earth's radius. This system is not equivalent to the ordinary Cartesian frame of reference as far as the directions of the axes vary, with the atmospheric particle motion from one point to the other. However, for the largescale processes in equations of thermo-hydrodynamic atmosphere the terms related to the spatial variations of the coordinate axes may be dropped in the first approximation (Pedlosky, 1979; Holton, 1975; Thompson, 1961). Therefore, the equation of motion in the spherical coordinate system (taking into account the relations between coordinates, mentioned above) has the same form as in the Cartesian frame of 
reference. This procedure simplifies the problem and investigation of dynamics of the large-scale processes in the atmosphere (Pedlosky, 1979; Holton, 1975; Thompson, 1961) and therefore, it will also be used for magnetoactive ionospheric medium.

The method of "frozen-in" coefficients in dynamic equations will also be used below. This method is known as the $\beta$-approximation (Gill, 1982; Pedlosky, 1979; Gossard and Hooke, 1975; Holton, 1975) in spherical hydrodynamics and meteorology. In this approximation the parameters $\omega_{0}(\theta), \nabla \omega_{0}(\theta), \mathrm{H}_{0}\left(\theta^{\prime}\right), \nabla \mathrm{H}_{0}\left(\theta^{\prime}\right)$ are constant at integration of dynamical equations, taking into account $\theta=\theta_{0}, \theta^{\prime}=\theta_{0}^{\prime}$. Medium motion is considered near $\theta_{0}$ and $\theta_{0}^{\prime}$, i.e. average values of adjunction of the geographical $\varphi_{0}$ and the geomagnetic $\varphi_{0}^{\prime}$ latitudes, respectively. In this case, dynamical equations transform into equations with constant coefficients, which may be investigated by the plane wave method. Application of $\beta$-approximation (or $\beta$-plane) leads to simple results, which gives the possibility to reveal more important features of motion on a rotating sphere, which differs from motion on a rotating plane. Furthermore we guess that a geographical latitude $\varphi$, coincides with a geomagnetic latitude $\varphi^{\prime}$ i.e. $\theta=\theta^{\prime}, \theta_{0}=\theta_{0}^{\prime}$.

Now we introduce the vectoral potential $\mathbf{h} /(\alpha \rho)=\nabla \times \mathbf{U}$, then we find:

$\nabla \times \frac{\mathbf{h}}{\alpha \rho}=\nabla \times \nabla \times \mathbf{U}=\nabla(\nabla \times \mathbf{U})-\Delta \mathbf{U}$.

Without losing generality, we can assume that $\nabla \times \mathbf{U}=0$. This is the Lorenz calibration condition, which guarantees a uniqueness of the solution for the vectoral potential $\mathbf{U}$. Seeking the solution of Eqs. (14) and (15) as a plane wave $\mathrm{V}, \mathrm{h} \sim \exp \{\mathrm{i}(\mathbf{k r}-\omega \mathrm{t})\}$, where $\mathbf{k}$ is the wave vector, $\omega$ is the frequency of perturbation. From Eq. (17) we obtain $\nabla \times \mathbf{h} /(\alpha \rho)=\mathrm{k}^{2} \mathbf{U}$. Taking into account this, Eqs. (14) and (15) may be written in the form:

$$
\begin{aligned}
& \frac{\partial}{\partial t} \nabla \times \mathbf{V}=\nabla \times \mathbf{V} \times 2 \omega_{0}-\nabla \times \mathbf{U} \times 2 \Omega_{H}, \\
& \frac{\partial}{\partial t} \nabla \times \mathbf{U}=\nabla \times \mathbf{V} \times 2 \Omega_{0}+\nabla \times \mathbf{U} \times 2 \Omega_{H},
\end{aligned}
$$

where $2 \Omega_{\mathbf{H}}=-\left(\mathrm{ck}^{2} /(4 \pi \mathrm{eN})\right) \mathbf{H}_{0}, 2 \Omega_{0}=\left(\mathrm{eN} /\left(\mathrm{N}_{\mathrm{n}} \mathrm{Mc}\right)\right) \mathbf{H}_{0}$. Equations (17) and (18) show that the electromagnetic waves must be generated by hydrodynamic and electromagnetic interaction on triplecomponent ionospheric plasma. From these equations follow that the changing of the velocity vortex $\nabla \times \mathbf{V}$ and the vectoral potential vortex $\nabla \times \mathbf{U}$ occurs under the action of Coriolis $\mathbf{F}_{\mathbf{C}}=\rho \mathbf{V} \times 2 \omega_{0}$ and the electromagnetic gyroscopic $\mathbf{F}_{H}=\rho \mathbf{V} \times 2 \Omega_{\mathbf{H}}, \mathbf{F}_{0}=\rho \mathbf{V} \times 2 \Omega_{0}$ forces. The solenoidal character automatically is taken into account by $\mathbf{V}$ and $\mathbf{U}$ vectors. Equations (18) and (19) represent a closed system of equations, describing the interaction of two uncompressible fluids, moving with velocities $\mathbf{V}$ and $\mathbf{U}$ under the action of the three gyroscopic forces mentioned above. In the general case, Eqs. (18) and (19) have a sixth order with respect to time and the corresponding dispersion equation has four nonzero roots for $\omega$ frequency. Two zero frequencies $(\partial / \partial t \sim \omega=0)$ correspond to hydrodynamic and electromagnetic equilibrium in the unperturbed state.

As far as $U$ has a velocity dimension $\mathrm{ms}^{-1}, \Omega_{H}$ and $\Omega_{0}$ have dimension $\mathrm{s}^{-1}$, differential Eq. (18) coincides with Eq. (19) replacing $\mathrm{V}$ by $\mathrm{U}$, and $\omega_{0}$ by $\Omega_{0}+2 \Omega_{H}$. The coincidence of these differential equations means that they must describe similar physical phenomena.

It must be mentioned that fundamental discoveries in quantum mechanics and different areas of theoretical physics are revealed by the analogy method. It will be shown below that the application of the analogy method gives the possibility to search the electromagnetic analogy of the atmospheric waves in the E- and F-regions of the ionosphere without solving Eqs. (18) and (19) in the general case. Let us consider a few particular cases for the system of Eqs. (18) and (19) for illustration.

1. For the E-region of the ionosphere $\Omega_{0} \ll \omega_{0}$ and dicussing perturbations for which $\Omega_{H} / \omega_{0} \ll|V| /|U| \ll \Omega_{H} / \Omega_{0}$ the first term in the right side of Eq. (18) exceeds the second one, but in Eq. (19) - vice versa. In this case formulas (18) and (19) give a closed system of equations for $\mathbf{V}$ and $\mathbf{U}$ vectors:

$$
\begin{aligned}
& \frac{\partial}{\partial t} \nabla \times \mathbf{V}=\nabla \times \mathbf{V} \times 2 \omega_{0}, \\
& \frac{\partial}{\partial t} \nabla \times \mathbf{U}=\nabla \times \mathbf{U} \times 2 \Omega_{H} .
\end{aligned}
$$

For small-scale $\left(\mathrm{L} \ll 10^{3} \mathrm{~km}\right)$ processes Eq. (20), well known in dynamical meteorology, has the general solution in the form of three-dimensional inertial waves, satisfying the dispersion Eq. (20) (Gill, 1982; Pedlosky, 1979; Gossard and Hooke, 1975):

$$
\omega=\omega_{\mathrm{I}}=\frac{1}{\mathrm{k}}\left(2 \omega_{0} \cdot \mathbf{k}\right)
$$

where $\mathrm{k}=\sqrt{\mathrm{k}_{\mathrm{x}}^{2}+\mathrm{k}_{\mathrm{y}}^{2}+\mathrm{k}_{\mathrm{z}}^{2}}$.

For large-scale ( $\left.\mathrm{L} \sim 10^{3}-10^{4} \mathrm{~km}\right)$ processes Eq. (20) has exact solution-slow planetary Rossby waves, satisfying the dispersion equation (Pedlosky, 1979; Gossard and Hooke, 1975; Khantadze, 1973):

$\omega=\omega_{R}=-\beta \frac{\mathrm{k}_{x}}{\mathrm{k}_{\mathrm{x}}^{2}+\mathrm{k}_{\mathrm{y}}^{2}}$,

where $\beta=\partial 2 \omega_{0 z} / \partial y=2 \omega_{0} \sin \theta_{0} / \mathrm{r}_{0}$ is the Rossby parameter, $\partial / \partial y=-\mathrm{r}_{0}^{-1} \partial / \partial \theta$. Here, $\theta_{0}$ is sum mean value of colatitude in the vicinity of which a motion in medium is considered.

Note once more that the Eq. (23) solution is obtained in a standard system of coordinates in the $\beta$-plane approximation (Gill, 1982; Pedlosky, 1979; Holton, 1975). 
The essence of this approximation is that at integration of vortex Eq. (20) parameters $2 \omega_{0 z}=2 \omega_{0} \cos \varphi_{0}$ and $\beta=\partial 2 \omega_{0 z} / \partial \mathrm{y}=2 \omega_{0} \sin \varphi_{0} / \mathrm{r}_{0}$ are considered as constants (after $(\nabla \times)$ operator action) and Eq. (20) is transformed into a differential equation with constant coefficients, of the solution of which may be obtained in the form of plane waves. This simple mean of the Earth's surface curvature calculation gives, at the same time, an opportunity to notice the most important features of the motions of the atmosphere on a rotating sphere, distinguishing it from a motion on a rotating plane. The same approximation is also used to obtain in the given work, a solution of equations of electromagnetic waves (21) and parameters of the geomagnetic field $\mathrm{H}_{0 z}=-2 \mathrm{H}_{p} \cos \theta_{0}$, $\mathrm{H}_{o y}=-\mathrm{H}_{p} \sin \theta_{0}$, and $\beta_{H}=\partial \mathrm{H}_{0 z} / \partial \mathrm{y}=-2 \mathrm{H}_{p} \sin \theta_{0} / \mathrm{r}_{0}$.

Equation (20) does not contain any more information about the new atmospheric waves. It is a cubic equation with respect to time and has nonzero own frequencies $\omega_{I}$ and $\omega_{R}$. Third root-zero frequency, as it is mentioned above, corresponds to a quasi-static and quasigeostrophic equilibrium $\left(\mathbf{V}_{g}=2 \omega_{0} \times \nabla P_{0} /\left(4 \rho \omega_{0}^{2}\right)\right.$ state of the atmosphere; $\mathrm{P}_{0}$ is the equilibrium pressure; $\mathrm{V}_{g}$ is velocity of geostrofic wind.

Using the analogy method, without solving Eq. (21) and applying only the expression (22), we can conclude that the analogy of the smallscale inertial waves in the ionosphere at the electromagnetic approach is the wellknown "atmospheric whistle" (helicons):

$\omega=\omega_{\mathrm{h}}=-\frac{1}{\mathrm{k}}\left(2 \Omega_{\mathbf{H}} \cdot \mathbf{k}\right)=\frac{\mathrm{ck}}{4 \pi \mathrm{eN}}\left(\mathbf{k} \cdot \mathbf{H}_{0}\right)$.

The sign "and" denotes the opposite directions of vectors $\omega_{0}$ and $\mathbf{H}_{0}$.

For largescale processes $\left(\mathrm{L} \sim 10^{3}-10^{4} \mathrm{~km}\right)$, when latitude variation of the geomagnetic field $\mathbf{H}_{0}$ is not negligible, electromagnetic analogy of Rossby waves (23) must exist in the E-region of the ionosphere (Kobaladze, et al., 1989; Khantadze, 2002):

$\omega=\omega_{\mathrm{H}}=-\beta_{\mathrm{H}} \frac{\mathrm{k}_{x}}{\mathrm{k}_{x}^{2}+\mathrm{k}_{y}^{2}}=\frac{\mathrm{c} \beta_{I}}{4 \pi \mathrm{eN}} \mathrm{k}_{x}$

where

$\beta_{\mathrm{H}}=\partial 2 \Omega_{\mathrm{Hz}} / \partial \mathrm{y}=-\mathrm{ck}^{2}\left(\partial \mathrm{H}_{0 \mathrm{z}} / \partial \mathrm{y}\right) /(4 \pi \mathrm{eN})=-\mathrm{c}$

$\left(\mathrm{k}_{\mathrm{x}}^{2}+\mathrm{k}_{\mathrm{y}}^{2}\right) \beta_{1} /(4 \pi \mathrm{eN}), \beta_{1}=\partial \mathrm{H}_{0 \mathrm{z}} / \partial \mathrm{y}=-2 \mathrm{H}_{\mathrm{p}} \sin \theta_{0} / \mathrm{r}_{0}$.

Taking into account both components of the geomagnetic field, we obtain (Khantadze, 1989; Aburjania et al., 2001):

$\omega_{\mathrm{H}}=\frac{\alpha}{4 \pi} \sqrt{\beta_{I}^{2}+\beta_{2}^{2}} \mathrm{k}_{x}=\frac{\mathrm{cH}_{p}}{4 \pi \mathrm{eN}} \frac{\sqrt{1+3 \sin ^{2} \theta}}{\mathrm{r}_{0}} \mathrm{k}_{x}$,

where $\beta_{2}=\partial \mathrm{H}_{0 y} / \partial \mathrm{y}$. This is the new mode of eigenoscillations of the E-region of the ionosphere.
Numerical calculations of the parameters of planetary waves (26) were carried out using models of the ionosphere and the neutral atmosphere (Jacchia, 1977) for low and high Sun activity. Numerical calculations show that at $\theta=45^{\circ}$ in the interval of heights $(90-150) \mathrm{km}$, phase velocity of waves $C_{H}=\omega_{H} / \mathrm{k}_{x}$ vary from 4 to $1.4 \mathrm{~km} \mathrm{~s}^{-1}$ at night, and from 400 to $800 \mathrm{~m} \mathrm{~s}^{-1}$ in the daytime. Periods $\left(\mathrm{T}_{H}=\lambda / \mathrm{C}_{H}\right.$ at $\left.\lambda=2 \times 10^{3} \mathrm{~km}\right)$ are in the interval of (1.5-6) $\mathrm{h}$ in the daytime and (4-12) min at night. Perturbation of the geomagnetic field of these waves $\mathrm{h}_{H}=\mathrm{H}_{p} \sqrt{1+3 \sin ^{2} \theta} \xi_{e} / \mathrm{r}_{0}$ (where $\xi_{e}$ is the electron displacement) is 8 and $80 \mathrm{nT}$ at $\xi_{e}=0.1 \mathrm{~km}$ and $\xi_{e}=1 \mathrm{~km}$. The influence of exosphere temperature on $\mathrm{C}_{H}$ and $\mathrm{T}_{H}$ is insignificant but is important for the magnetic field perturbations. $\mathrm{C}_{H}$ and $\mathrm{T}_{H}$ values are substantially different in the daytime and at night as far as electron concentration in the E-region of the ionosphere varies by magnitude with order during a given day.

These oscillations were observed experimentally (Bauer et al., 1995; Sharadze et al., 1988) at middle latitudes of the E-region of the ionosphere and were extracted as middle-latitude long-period oscillations. But it is evident from Eq. (26), that there aren't any restrictions for the existence of these perturbations at both high and low latitudes. They are revealed especially by a worldwide network of ionospheric and magnetospheric observatories during earthquakes, magnetic storms and artificial explosions (Hayakawa, 1999; Hajkovicz, 1991; Al'perovich et al., 1985).

Equation (21) does not contain any additional information. Now we demonstrate this in general case for high and moderate latitudes $\left(\mathbf{H}_{0} \approx \mathrm{H}_{0 z} \mathbf{e}_{z}\right)$. Let us write the expression (21) in the following form:

$\omega \mathbf{k} \times \mathbf{U}=\mathrm{i} 2 \Omega_{H z} \mathrm{k}_{z} \mathbf{U}-\beta_{H} \mathrm{U}_{y} \mathbf{e}_{z}$,

$\mathbf{k} \cdot \mathbf{U}=0$.

Multiplying Eq. (27) by k vectorially and using Eq. (28) we obtain:

$\mathbf{U}=-\mathrm{i} \frac{2 \Omega_{H z}}{\omega \mathrm{k}^{2}} \mathrm{k}_{z} \mathbf{k} \times \mathbf{U}+\frac{\beta_{H}}{\omega \mathrm{k}^{2}} \mathrm{U}_{y} \mathbf{k} \times \mathbf{e}_{z}$.

Excluding the expression $\mathbf{k} \times \mathbf{U}$ using Eq. (27) and taking into account $\left(\mathbf{k} \times \mathbf{e}_{z}\right)_{x}=\mathrm{k}_{y}, \quad\left(\mathbf{k} \times \mathbf{e}_{z}\right)_{y}=-\mathrm{k}_{x}$, $\left(\mathbf{k} \times \mathbf{e}_{\mathbf{z}}\right)_{z}=0$ from Eq. (29), we obtain the system of equations for $\mathrm{U}_{x}, \mathrm{U}_{y}$, and $\mathrm{U}_{z}$ component

$\left(1-\frac{\omega_{h}^{2}}{\omega^{2}}\right) \mathrm{U}_{x}=\frac{\beta_{H}}{\omega \mathrm{k}^{2}} \mathrm{k}_{y} \mathrm{U}_{y}$,

$\left(1-\frac{\omega_{h}^{2}}{\omega^{2}}\right) \mathrm{U}_{y}=-\frac{\beta_{H}}{\omega \mathrm{k}^{2}} \mathrm{k}_{x} \mathrm{U}_{y}$, 
$\left(1-\frac{\omega_{h}^{2}}{\omega^{2}}\right) \mathrm{U}_{z}=\mathrm{i} \frac{2 \Omega_{H z} \mathrm{k}_{z}}{\omega \mathrm{k}^{2}} \beta_{H} \mathrm{U}_{y}$.

It follows from Eq. (31) that

$$
\left(1-\frac{\omega_{h}^{2}}{\omega^{2}}+\frac{\beta_{H} \mathrm{k}_{x}}{\omega \mathrm{k}^{2}}\right) \mathrm{U}_{y}=0,
$$

where $\omega_{h}=\mathrm{ckk}_{z} \mathrm{H}_{0 z} /(4 \pi \mathrm{eN})$. So, we have two cases: $\mathrm{U}_{y} \neq 0$ or $\mathrm{U}_{y}=0$.

a) At $\mathrm{U}_{y} \neq 0$ in Eq. (33) round bracket tends to zero

$$
\begin{aligned}
& \left(1-\frac{\omega_{h}^{2}}{\omega^{2}}+\frac{\beta_{H} \mathrm{k}_{x}}{\omega \mathrm{k}^{2}}\right)=0, \quad \text { or } \\
& \left(1-\frac{\omega_{h}^{2}}{\omega^{2}}\right)=\frac{\beta_{H} \mathrm{k}_{x}}{\omega \mathrm{k}^{2}} .
\end{aligned}
$$

Substituting this expression into Eq.D (30), we obtain:

$$
-\frac{\beta_{H} \mathrm{k}_{x}}{\omega \mathrm{k}^{2}} \mathrm{k}_{x} \mathrm{U}_{x}=\frac{\beta_{H}}{\omega \mathrm{k}^{2}} \mathrm{k}_{y} \mathrm{U}_{y} .
$$

From this it follows that $\mathrm{k}_{x} \mathrm{U}_{x}+\mathrm{k}_{y} \mathrm{U}_{y}=0$. Taking into account Eq. (28) $\mathrm{k}_{x} \mathrm{U}_{x}+\mathrm{k}_{y} \mathrm{U}_{y}+\mathrm{k}_{z} \mathrm{U}_{z}=0$, we obtain $\mathrm{k}_{z} \mathrm{U}_{z}=0$. Therefore, if $\mathrm{k}_{z}=0$, from formula (32) yields $\mathrm{U}_{z}=0$. Thus, $\mathrm{k}_{z}, \mathrm{U}_{z}$ and $\omega_{h}$ vanish simultaneously at $\mathrm{U}_{y} \neq 0$. If $\mathrm{U}_{x}$ and $\mathrm{U}_{y}$ are nonzero, in this case the dispersion Eq. (34) gives only a Rossby wave analogy

$$
\omega=\omega_{H}=-\beta_{H} \frac{\mathrm{k}_{x}}{\mathrm{k}_{x}^{2}+\mathrm{k}_{y}^{2}}=\frac{\mathrm{c} \beta_{I}}{4 \pi \mathrm{eN}} \mathrm{k}_{x}
$$

b) Left side of Eqs. (30) and (32) tends to zero at $\mathrm{U}_{y}=0$

$$
\left(1-\frac{\omega_{h}^{2}}{\omega^{2}}\right) \mathrm{U}_{x}=0 ; \quad\left(1-\frac{\omega_{h}^{2}}{\omega^{2}}\right) \mathrm{U}_{z}=0 .
$$

If $\mathrm{U}_{x}=0, \mathrm{U}_{z}=0$, we have a trivial zero solution, which corresponds to the equilibrium state, when electric drift velocity and geostrophic wind velocity are equal (Gershman, 1974). At $\mathrm{U}_{y} \neq 0$ and $\mathrm{U}_{z} \neq 0$ we obtain the dispersion equation for helicons $\omega=\omega_{h}=\mathrm{ckk}_{z} \mathrm{H}_{0 z} /(4 \pi \mathrm{eN})$.

Analysis of the possible solutions of Eq. (21) shows that the electromagnetic planetary wave $\mathrm{C}_{H}=\omega_{H} / \mathrm{k}_{x}$ is the unique solution of Eq. (21) at $U_{y} \neq 0$, for which helicons are automatically excluded. But at $\mathrm{U}_{y}=0$ helicons are a unique solution, and $\mathrm{C}_{H}$-waves are filtered out. By the analogy method this proof can be carried out directly for inertial and planetary Rossby waves, i.e. for Fridman's equation (20).
2. In the F-region of the ionosphere $\Omega_{0} \gg \omega_{0}$ and discussing perturbations for which $\omega_{H} / \Omega_{0} \ll|V| /|U| \ll \Omega_{H} / \omega_{0}$, the first term can be neglected in comparison with the second one in the right side of Eq. (18) and vice versa in Eq. (19):

$\frac{\partial}{\partial t} \nabla \times \mathbf{V}=-\nabla \times \mathbf{U} \times 2 \Omega_{H}$,

$\frac{\partial}{\partial t} \nabla \times \mathbf{U}=\nabla \times \mathbf{V} \times 2 \Omega_{0}$.

It is easy to show that the system of Eqs. (35) and (36) does not contain the Hall parameter $\alpha$ and therefore, this system can be applied to the investigation of electromagnetic processes in the F-region of the ionosphere.

For small-scale processes, when latitude variations of the geomagnetic field $\mathrm{H}_{0}$ can be neglected, parameters $2 \Omega_{H}$ and $2 \Omega_{0}$ become constant and the system of Eqs. (35) and (36) can be solved in the general case. Actually, using transversal condition of the waves $(\mathbf{k} \cdot \mathbf{U}=0$, $(\mathbf{k} \cdot \mathbf{V})=0$ from Eqs. (35) and (36) we obtain:

$\omega \mathbf{k} \times \mathbf{V}=-\mathrm{i}\left(\mathbf{k} \cdot 2 \Omega_{\mathbf{H}}\right) \mathbf{U} ; \omega \mathbf{k} \times \mathbf{U}=\mathrm{i}\left(\mathbf{k} \cdot 2 \Omega_{0}\right) \mathbf{V}$.

Eliminating $U$ and $V$ from these expressions, we obtain a dispersion equation for modified Alfvén waves in the F-region of the ionosphere:

$\omega^{2}=-\frac{1}{\mathrm{k}}\left(\mathbf{k} \cdot 2 \Omega_{0}\right) \cdot \frac{1}{\mathrm{k}}\left(\mathbf{k} \cdot 2 \Omega_{\mathbf{H}}\right)$

from which follows:

$\omega_{1,2}= \pm \frac{\sqrt{\eta}}{\sqrt{4 \pi \mathrm{MN}}}\left(\mathbf{k} \cdot \mathbf{H}_{\mathbf{0}}\right)= \pm \sqrt{\eta} \omega_{\mathrm{A}}$.

Here, the nondimensional parameter $\eta=\mathrm{N} / \mathrm{N}_{n}$ denotes the degree of plasma ionization; $\omega_{\mathrm{A}}=\left(\mathbf{k} \cdot \mathbf{H}_{0}\right) / \sqrt{4 \pi \mathrm{MN}}$ is the Alfvén frequency. Modified Alfvén waves are slow waves as far as the parameter $\eta$ varies in the interval $10^{-7}-10^{-3}$ for F-region of the ionosphere at (200$500) \mathrm{km}$. Dispersion Eq. (38) has two roots for positive and negative propagation directions. Group velocity of these perturbations is directed along the force lines of the geomagnetic field $\mathbf{H}_{0}$.

Similarly to Eq. (38), from Eqs. (35) and (36) we obtain only one root for the dispersion equation, describing the propagation of zonal perturbations along latitude circles (along xaxis directing towards parallel) for large-scale processes, when latitude variations of the geomagnetic field is not negligible (Khantadze, 2002; Aburjania et al., 2002):

$\omega=\omega_{n}=\sqrt{\eta} \frac{\mathrm{H}_{p}}{\sqrt{4 \pi \mathrm{MN}}} \frac{\sqrt{1+3 \sin ^{2} \theta}}{\mathrm{r}_{0}}$. 
Calculations show that phase velocity of these waves $\mathrm{C}_{n}=\omega_{n} / \mathrm{k}_{x}$ are in the range of $(20-1400) \mathrm{km} \mathrm{s}^{-1}$ at a height of $(200-500) \mathrm{km}$, the wavelength is $\lambda=2 \times 10^{3} \mathrm{~km}, \theta=45^{\circ}$, the exosphere temperature is $\mathrm{T}_{\text {exos }}=600^{\circ} \mathrm{K}$; and $(10-50) \mathrm{km} \mathrm{s}^{-1}$ at $\mathrm{T}_{\text {exos }}=2600^{\circ}$. The period of these waves $\mathrm{T}_{n}=2 \pi / \omega_{n}$ does not depend on a wavelength and is in the interval (105-3) $\mathrm{s}$ at $\mathrm{T}_{\text {exos }}=600^{\circ} \mathrm{K}$ and $(210-40) \mathrm{s}$ at $\mathrm{T}_{\text {exos }}=2600^{\circ}$. Magnetic pulsations, induced by these waves have the same order by magnitude as $\mathrm{C}_{H}$-waves, $h_{n} \approx h_{H}$. The coriolis force and the existence of the ordinary Rossby waves in the F-region of the ionosphere leads to the dispersion relation $\left(\omega / \mathbf{k}_{x}\right)^{2}=C_{n}^{2}\left(1-\omega_{R} / \omega\right)^{-1}$. Periods, phase velocities and amplitudes of geomagnetic pulsations for $\mathrm{C}_{n}$-waves in a middle-latitude ionosphere are in agreement with observation data of both middle-latitude and large-scale electromagnetic perturbations, generated in F-region of the ionosphere at powerful earthquakes and magnetic storms (Hayakawa, 1999; Bauer et al., 1995; Hajkowicz, 1991).

3. Now we consider the frequency band $\omega \ll 2 \Omega_{H}$. The left side of Eq. (19) may be neglected in comparison with the free terms (which is fulfilled for potential electric fields):

$\frac{\partial}{\partial t} \nabla \times \mathbf{V}=\nabla \times \mathbf{V} \times 2 \omega_{0}-\nabla \times \mathbf{U} \times 2 \Omega_{H}$,

$0=\nabla \times \mathbf{V} \times 2 \Omega_{0}+\nabla \times \mathbf{U} \times 2 \Omega_{H}$.

Eliminating $\nabla \times \mathbf{U} \times 2 \Omega_{H}$, we obtain the generalized Fridman's equation for vorticity:

$$
\begin{gathered}
\frac{\partial}{\partial t} \nabla \times \mathbf{V}=\nabla \times\left(2 \omega_{0}+2 \Omega_{0}\right)= \\
\nabla \times \mathbf{V} \times 2\left(\omega_{0}+\eta \omega_{i}\right),
\end{gathered}
$$

where $\omega_{i}$ is the ion gyrofrequency.

From Eq. (42), as in case 1), it follows, that two classes of the waves must be generated: the smallscale modified inertial waves with frequency

$\omega=\omega_{\mathrm{I}}^{\prime}=\frac{1}{\mathrm{k}}\left(2 \omega_{0}^{\prime} \cdot \mathbf{k}\right)$.

where $2 \omega_{0}^{\prime}=2\left(\omega_{0}+\eta \omega_{i}\right)$ and the largescale planetary Rossbytype waves having both a hydrodynamic and an electromagnetic nature (compare with Tolstoy, 1967; Khantadze, 1967):

$\omega=\omega_{R}^{\prime}=-\beta^{\prime} \frac{\mathrm{k}_{x}}{\mathrm{k}_{x}^{2}+\mathrm{k}_{y}^{2}}$.

Here, $\beta^{\prime}=\beta+\beta_{i}, \beta_{i}=\eta \partial \omega_{i z} / \partial \mathrm{y} . \quad$ Calculations show that phase velocities of $\mathrm{C}_{R}^{\prime}=\omega_{R}^{\prime} / \mathrm{k}_{x}=-\beta^{\prime} \lambda^{2} /\left(4 \pi^{2}\right)$ waves are in the range of $(-2-+80) \mathrm{m} \mathrm{s}^{-1}$ in the daytime, at the heights of $(90-150) \mathrm{km} \mathrm{T}_{\text {exos }}=600^{\circ} \mathrm{K}$ and $\lambda=2 \times 10^{3} \mathrm{~km}$. For $\lambda=2 \times 10^{4} \mathrm{~km}$ phase velocities vary from $\left(-41 \mathrm{~m} \mathrm{~s}^{-1}\right)$ to $\left(+1,8 \mathrm{~km} \mathrm{~s}^{-1}\right)$ in the daytime and in the range of $(-41-11) \mathrm{m} \mathrm{s}^{-1}$ at night. Velocities change from $\left(3 \mathrm{~m} \mathrm{~s}^{-1}\right.$ to $+60 \mathrm{~m} \mathrm{~s}^{-1}$ in the daytime and from $\left(2 \mathrm{~m} \mathrm{~s}^{-1}\right)$ to $\left(1.3 \mathrm{~m} \mathrm{~s}^{-1}\right)$ at night, $\mathrm{T}_{\text {exos }}=2600^{\circ} \mathrm{K}$ and $\lambda=2 \times 10^{3} \mathrm{~km}$. In this case the sign "and" points in the direction of the phase velocity from the east to the west, sign " + " from the west to the east. Calculations show that $\beta^{\prime}=\left(\mathrm{N} \omega_{i z} / \mathrm{N}_{n}-\Omega_{0}\right) 2 \sin \theta / \mathrm{r}_{0}$ tends to zero and $\mathrm{C}_{R}^{\prime}=0$ in the daytime at the height of $115 \mathrm{~km}$. Parameter $\beta^{\prime}$ tends to zero at the height $150 \mathrm{~km}$ of the nightly ionosphere. Hence, ordinary slow planetary Rossby waves, moving from the west to the east direction in the daytime, prevailed in the lower E-region at the heights of $(90-115) \mathrm{km}$; fast planetary waves, having an electromagnetic nature and moving from the west to the east direction, will prevail higher of critical altitudes. The Hall region is completely occupied by the slow Rossby waves at the nightly ionosphere. Hence, magnetic control of planetary waves in the ionosphere depends on critical altitude, where the condition $\beta^{\prime}=\beta+\beta_{i}=0$ is fulfilled. These altitudes experimentally may be revealed at the registration of planetary waves jointly by both ionospheric and magnetosphere observatories. Calculation shows, that periods $\mathrm{T}_{R}^{\prime}=2 \pi / \omega_{R}^{\prime}$ are in the interval from 14 day's to $8 \mathrm{~h}$ at the heights $(90$ $150) \mathrm{km}, \mathrm{T}_{\text {exos }}=600^{\circ} \mathrm{K}, \lambda=2 \times 10^{3} \mathrm{~km}$. $\mathrm{T}_{R}^{\prime}$ vary from 14 days to $2 \mathrm{~h}$ at $\mathrm{T}_{\text {exos }}=2600^{\circ} \mathrm{K}$. Perturbation of the geomagnetic field $\mathrm{h}_{R}=\approx\left|4 \pi \mathrm{eNC}_{R}^{\prime} \xi\right| / \mathrm{c}$ runs up to a few tens of $\mathrm{nT}$, where $\xi$ is the ion(neutral) displacement. $\mathrm{Pa}$ rameters of $\mathrm{C}_{R}^{\prime}$-waves are correlated well with observable parameters of planetary electromagnetic waves in the E-region of the ionosphere at moderate latitudes at any season of the year (Sharadze et al., 1989; Cavalieri et al., 1976; 1974).

Vortex-free flow $\nabla \times \mathbf{V}=0$ and other cases, depending on orientation of both $\mathbf{V}$ and $\mathbf{U}$ velocities with respect to the geomagnetic field $\mathbf{H}_{0}$ in the ionosphere, may be considered easily, but these problems are not considered in this paper.

\section{Conclusion}

The analogy method yields the simple and important physical results. Particularly, investigation of Eqs. (18) and (19) show that four normal modes: small-scale inertial waves, atmospheric whistles (helicons), fast large-scale electromagnetic planetary $\mathrm{C}_{H}=\omega_{H} / \mathrm{k}_{x}$-waves and slow Rossby-type waves must exist in the Eregion of the ionosphere. Modified smallscale slow Alfvén waves with $\omega_{+}$and $\omega_{-}$frequencies, fast large-scale electromagnetic planetary waves $\mathrm{C}_{n}=\omega_{n} / \mathrm{k}_{x}$ and ordinary slow planetary Rossby waves must be generated in the F-region of the ionosphere. Two eigen-frequencies, $\omega=0$ also have the physical notion and correspond to hydrodynamic and electromagnetic equilibrium state of the ionospheric medium in a background state, where geostrophycal wind velocity coincides with electric drift velocity. 
Existence of the large-scale fast waves $\mathrm{C}_{H}$ (in E-region), $\mathrm{C}_{n}$ (in F-region) and slow Rossby-type planetary waves $\mathrm{C}_{R}^{\prime}$ (in both the E- and F-regions) are caused by the inhomogeneity of the geomagnetic field $\mathbf{H}_{0}$. Slow waves are generated by the polarized electrostatic dynamo field of polarization $\left(\mathbf{E}_{d}=\mathbf{V} \times \mathbf{H}_{0} / \mathrm{c}\right)$, fast waves - by the vortex electric field $\mathbf{E}_{V}=\mathbf{V}_{D} \times \mathbf{H}_{0} / \mathrm{c}$. The frequencies of these waves vary in the band $\omega \sim 1-10^{-6} \mathrm{~s}^{-1}$ and occupy both infrasound and ultra-low frequency (ULF) bands. The wavelength is $\lambda \sim\left(10^{3}-10^{4}\right) \mathrm{km}$, the period of oscillation is $\mathrm{T} \sim 1 \mathrm{~s}-14$ days. Waves generate pulsations of geomagnetic field $\left(1-10^{2}\right) \mathrm{nT}$.

Dynamics of the slow planetary electromagnetic waves in the ionosphere are studied experimentally, more or less. Experimental investigation of features of the fast largescale electromagnetic waves must be realized. Formulas (26) and (39) show that fast electromagnetic large-scale $\left(\mathrm{L}\left(10^{3}-10^{4}\right) \mathrm{km}\right)$ atmospheric waves both in the E- and Fregions of the ionosphere have a general-planetary character and occupy latitudes from the pole $(\theta=0)$ to the equator $(\theta=\pi / 2)$.

The fast electromagnetic atmospheric waves at ionospheric altitudes can be experimentally revealed and registered using their specific features:

1. A wide range of phase velocity dependence on latitude (phase velocities of these waves are increased from the pole to the equator; they are doubled at the equator).

2. A high variation (by magnitude) of electron concentration $\mathrm{N}$ substantially increases the phase velocity of $\mathrm{C}_{H}=\omega_{H} / \mathrm{k}_{x}$-waves in the E-region of the ionosphere at nightly conditions (from a few hundreds $\mathrm{m} / \mathrm{s}$ in the daytime to a few tens of $\mathrm{km} / \mathrm{s}$ at night).

3. Application of the well-known profiles $\mathrm{N}(\mathrm{h})$ allows us to calculate uniquely the height distribution of the $\mathrm{C}_{H}$-waves in the E-region of the ionosphere and, conversely, from a height distribution of $\mathrm{C}_{H}(\mathrm{~h})$-waves we can plot the dependence of the concentration $\mathrm{N}(\mathrm{h})$ on an altitude.

4. Altitude variation of the neutral component concentration $\mathrm{N}_{n}(\mathrm{~h})$ leads to a strong increase in the phase velocity of the $\mathrm{C}_{n}$-waves (phase velocity of $\mathrm{C}_{n}$-waves at heights of (200-500) km is increased from a few km s${ }^{-1}$ up to $1000 \mathrm{~km} \mathrm{~s}^{-1}$ ) in the F-region of the ionosphere.

5. The response of especially the $\mathrm{C}_{H^{-}}$and $\mathrm{C}_{n}$-waves at earthquakes, magnetic storms, artificial explosions and magnetic activity of the Sun.

6. Registeration of electromagnetic and large-scale $\left(10^{3}-\right.$ $10^{4} \mathrm{~km}$ ) character of both the $\mathrm{C}_{H}$ and $\mathrm{C}_{n}$-waves by a world-wide network of ionospheric and magnetospheric observatories.

In conclusion we can say that planetary waves in the ionosphere, unlike the troposphere, generate high temporalvarying "weather". Waves occupy a large temporal interval from two days or more (slow planetary $\omega_{R}^{\prime}$-waves) and from a few hours up to a few minutes or less (fast planetary $\omega_{H}$ and $\omega_{n}$-waves).

Acknowledgements. The research described in this publication was made possible in part by Award No. 3317 of the Georgian Research \& Development Foundation (GRDF) and the U. S. Civilian Research \& Development Foundation for the Independent State of the Former Soviet Union (CRDF).

Topical Editor M. Lester thanks two referees for their help in evaluating this paper.

\section{References}

Aburjania, G. D., Khantadze,A. G., and Kharshiladze, O. A.: Nonlinear electromagnetic planetary vortex structures in F-region of the ionosphere, Plasma Phys. Rep., 28, 633-638, 2002.

Aburjania, G. D. and Khantadze, A. G.: Long-scale electromagnetic wavy structures in E-region of the ionosphere, Geomag. Aeron., 42, 245-251, 2002.

Aburjania, G. D., Jandieri, G. V., and Khantadze, A. G.: Nonlinear evolution of planetary-scale electromagnetic waves in E-region of the ionosphere for a spherical Earth model, Applied Mathematics and Informatics, 6, 1-24, 2001.

Alfvén, H. and Falthammar, C.-G.: Cosmical electrodynamics, Clarendon Press, Oxford, 1963).

Al'perovich, L. S., Ponomarev, E. A., and Fedorovich, G. V.: Geophysical phenomena modelling by an explosion: a review, Izv. Phys. Solid Earth, 21, 816-825, 1985.

Bauer, T. M., Baumjohann, W., Treumann, R. A., Svopka, N., and Luhr, H.: Low- frequency waves in the near-Earth plasma sheet, J. Geophys. Res., 100, 9605-9617, 1995.

Cowling, T. C.: Magnetohydynamics, Adam Higer Ltd., 1975.

Cavalieri, D. J., Deland, R. J., Potemra, T. A., and Gavin, R. F.: The correlation of VLF propagation variations with atmospheric planetary-scale waves, J. Atmosph. Terr. Phys., 36, 561-574, 1974.

Cavalieri, D. J.: Traveling planetary-scale waves in the E-region, J. Atmosph. Terr. Phys., 38, 965-977, 1976.

Gershman, B. I.: Dynamics of the ionospheric plasma, Nauka, Moscow, 1974.

Gill, A. E.: Atmosphere-ocean dynamics, Academic Press, London, 1982.

Gossard, E. E. and Hooke, W. H.: Waves in the Atmosphere, Elsevier Sci. Publ. Comp., Amsterdam-Oxford-New York, 1975.

Hayakawa, M.: Atmospheric and ionospheric electromagnetic phenomena associated with earthquakes, edited by Hayakawa, M. Terra Sci. Pub. Co., Tokyo, 1999.

Hajkowicz, L. A.: Global onset and propagation of large-scale traveling ionospheric disturbanced as a result of the great storm of 13 March 1989, Planet Space Sci., 39, 583-593, 1991.

Holton, J. R.: The dynamic meteorology of the stratosphere and mesosphere, Amer. Meteor. Soc., Boston, Massachusetts, 1975.

Jacchia, L. G.: Thermospheric temperature, density and composition: new models, Spec. Rep. Smithsonian Astrophys. Obser., 375, 1-106, 1977.

Khantadze, A. G.: Electromagnetic planetary waves in the Earth ionosphere, Gemagn. Aeron., 42, 333-335, 2002.

Khantadze, A. G.: Hydromagnetic gradient waves in dynamoregion of the ionosphere, Bull. Acad. Sci. Georgian SSR, 123 69-71, 1986. 
Khantadze, A. G.: On the dynamics of conductive atmosphere, Nauka, Tbilisi, 1973.

Khantadze, A. G.: Determination of the wind field by the pressure gradient field and latitudinal effect of geomagnetic field, Proc. Inst. Geophys. Acad. Sci. Georgian SSR, 9, 112-116, 1967.

Kobaladze, Z. L. and Khantadze, A. G.: On the propagation of large-scale disturbances in the ionosphere, Bull. Acad. Sci. Georgian SSR, 134, 97-100, 1989.

Pedlosky, J.: Geophysical fluid dynamics, Springer-Verlag, New York, 1979.

Sharadze, Z. S., Japaridze, G. N., Kikvilashvili, G. B., Liadze, Z. L., Matiashvili, T. G., and Mosashvili, N. B.: Wave disturbaces of nonacoustical nature in the midlatitude ionosphere, Geomag. Aeron., 28, 446-451, 1988.
Sharadze, Z. S., Mosashvili, N. B., Pushkova, G. N., and Judovich, L. A.: Long-period wave disturbances in E-region of the ionosphere, Geomag. Aeron., 29, 1032-1035, 1989.

Thompson, P. D.: Numerical weather analysis and prediction, The Macmillan Company, New York, 1961.

Tolstoy, I.: Hydromagnetic waves in the ionosphere, J. Geophys. Res., 7, 1435-1442, 1967.

Wait, J. R.: Electromagnetic waves in stratified media, Pergamon Press, Oxford, 1962. 